\title{
Artificial Intelligence in the Management of Intracranial Aneurysms: Current Status and Future Perspectives
}

\author{
(D) Z. Shi, (D) B. Hu, (D).J. Schoepf, (D) R.H. Savage, (D) D.M. Dargis, (D) C.W. Pan, (D)X.L. Li, (D) Q.Q. Ni, (D) G.M. Lu, and (D) L.J. Zhang
} -

\begin{abstract}
SUMMARY: Intracranial aneurysms with subarachnoid hemorrhage lead to high morbidity and mortality. It is of critical importance to detect aneurysms, identify risk factors of rupture, and predict treatment response of aneurysms to guide clinical interventions. Artificial intelligence has received worldwide attention for its impressive performance in image-based tasks. Artificial intelligence serves as an adjunct to physicians in a series of clinical settings, which substantially improves diagnostic accuracy while reducing physicians' workload. Computerassisted diagnosis systems of aneurysms based on MRA and CTA using deep learning have been evaluated, and excellent performances have been reported. Artificial intelligence has also been used in automated morphologic calculation, rupture risk stratification, and outcomes prediction with the implementation of machine learning methods, which have exhibited incremental value. This review summarizes current advances of artificial intelligence in the management of aneurysms, including detection and prediction. The challenges and future directions of clinical implementations of artificial intelligence are briefly discussed.
\end{abstract}

ABBREVIATIONS: $\mathrm{AI}=$ artificial intelligence; $\mathrm{AUC}=$ area under the curve; $\mathrm{CAD}=$ computer-assisted diagnostics; $\mathrm{DL}=\mathrm{deep}$ learning; $\mathrm{FP}=\mathrm{false-positive;}$ $\mathrm{ML}=$ machine learning; SVM = support vector machines

U nruptured intracranial aneurysm remains a major public health concern affecting about $3 \%-7 \%$ of the general population. ${ }^{1,2}$ CTA and MRA are the preferred techniques to identify aneurysms. A steadily increasing number of radiologic examinations are performed for neurologic diagnoses, which require human expertise in image interpretation. However, experienced radiologists are in a relative shortage due to an ever-increasing demand for imaging studies. ${ }^{3}$ The excess workload imposed on physicians requires them to interpret 1 image every 3-4 seconds within work hours. As a result, uncertainty and inevitable errors when making diagnoses and decisions may arise. ${ }^{4}$

Received September 30, 2019; accepted after revision December 16. From the Department of Medical Imaging (Z.S., B.H., Q.Q.N., G.M.L., L.J.Z.), Jinling Hospital, Medical School of Nanjing University, Nanjing, Jiangsu, China; Division of Cardiovascular Imaging (U.J.S., R.H.S., D.M.D.), Department of Radiology and Radiological Science, Medical University of South Carolina, Charleston, South Carolina; DeepWise AI Lab (C.W.P., X.L.L.), Beijing, China; and Peng Cheng Laboratory (X.L.L.), Vanke Cloud City Phase I, Nanshan District, Shenzhen, Guangdong, China.

This work was supported by the National Key Research and Development Program of China (2017YFC0113400 for L.J.Z.) and Key Projects of the National Natural Science Foundation of China (81830057 for L.J.Z.).

Please address correspondence to Long Jiang Zhang, MD, PhD, Department of Medical Imaging, Jinling Hospital, Medical School of Nanjing University, Nanjing, Jiangsu, 210002, China; e-mail: kevinzhlj@163.com

\section{- Indicates open access to non-subscribers at www.ajnr.org}

Indicates article with supplemental on-line table.

http://dx.doi.org/10.3174/ajnr.A6468
As the most common cause of nontraumatic SAH, aneurysms are responsible for $85 \%$ of SAH cases. The overall annual rupture risk is $0.95 \%$ and is associated with a high risk of morbidity and mortality. ${ }^{5}$ Endovascular and surgical treatments have a $3 \%-10 \%$ risk of stroke or death and may not permanently eliminate the propensity for rupture. ${ }^{6}$ Therefore, recognition of risk factors and construction of prediction models related to aneurysm initiation, growth, rupture, and assessment of intervention have been popular areas of investigation. However, the management of aneurysms is associated with multiple challenges in all aspects of clinical practice, which cannot be overcome easily by conventional methodology.

Artificial intelligence (AI) describes the development of computer algorithms that simulate human intelligence, which includes learning, reasoning, and self-correction. Brilliant progress has been made that allows machines to automatically interpret complicated data as part of routine clinical care. ${ }^{7}$ AI is also expected to satisfy the clinician's need for higher accuracy and better efficacy at all stages of aneurysm management. This review summarizes current applications of $\mathrm{AI}$ in the various phases (Fig 1) of intracranial aneurysm management, which facilitates optimized workflows and adds benefit to patient care. In addition, this review discusses the future challenges and directions of AI-based management of aneurysm. 


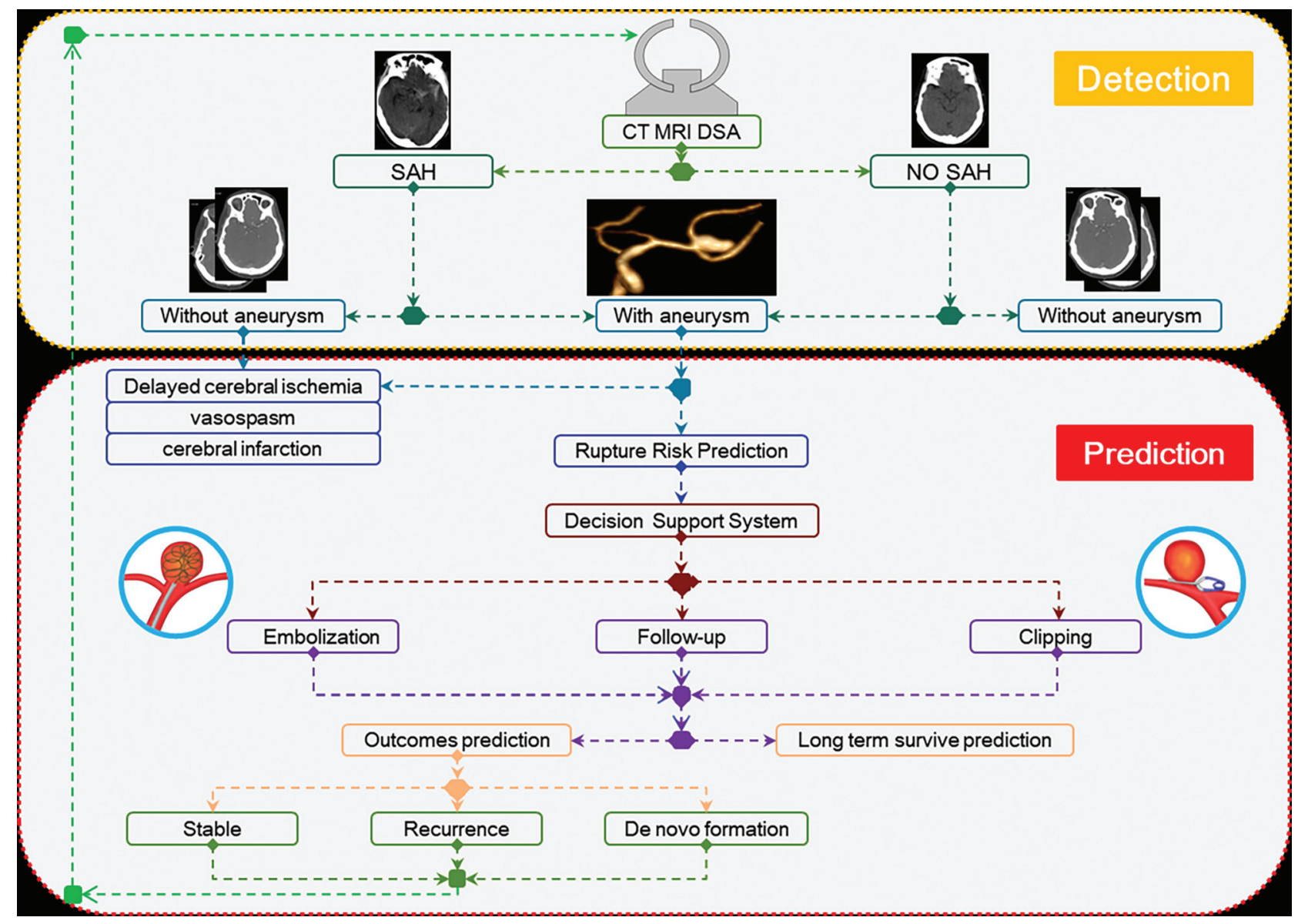

FIG 1. Various phases of intracranial aneurysm management. They include the procedures of detection and prediction.

\section{Brief Overview of Al}

AI can perform tasks with humanlike intelligence such as pattern recognition, object identification, and problem resolution. AI can make autonomous decisions based on the data collected for training. Similarly, in the realm of medicine, AI can identify a likely diagnosis and select a suitable treatment based on health records or imaging information without any explicit programming. Machine learning (ML) endows AI with the ability to learn and train models to extract and memorize features and related parameters. There are 3 types of ML: supervised (training with specific labels or annotations), unsupervised (training without specific labels and the algorithm clusters data to reveal underlying patterns), and semi- or weakly supervised learning (training with both labeled and unlabeled data to reduce the annotation burden). ${ }^{8}$

Deep learning (DL) is a subset of machine learning that is end-to-end ML in which it receives input and learns its salient features without explicit examples. ${ }^{9}$ There are 2 key requirements for ML: 1) Data are relevant and detailed enough for answering the clinical questions; and 2) a computational ML technique is appropriate for the type, amount, and complexity of the available data. Finally, it is necessary to further validate the usefulness of ML models in real-world practice. ${ }^{10}$

Traditional ML algorithms predefine engineered features that can describe the patterns inherent in regions of interest with explicit parameters based on expert knowledge. Support vector machines (SVM) and random forests are classic ML techniques.
These algorithms have been successfully used in AI studies. ${ }^{11,12}$ Compared with traditional ML algorithms, DL uses an artificial neural network that extracts features from images automatically to create its own filters, called feature maps (independent of human input) and memorizes visual patterns with highest frequency. There are convolution layers, pooling layers, fully connected layers, and normalization layers; the pooling reduces the number of parameters and reduces overfitting. Once a final output is made that is compared with ground truth, the model will reweight the inputs until the best performance is reached, which can be repeated many times (called epochs). For feature-extraction and selection, DL algorithms do not need to predefine features, and the feature representations can be directly learned by navigating the data space. Most DL networks fundamentally build on some basic and similar neural network blocks or layers based on DL attempts to model high-level abstractions. ${ }^{13}$ Therefore, the extracted complex features are then fed through the last layer of the network for the target, such as classification or prediction. Convolutional neural networks are typical types of DL architecture, which consist of characteristic layers of convolutional operations on the input images to extract abstract features. ${ }^{13}$

\section{Computer-Assisted Diagnosis of Aneurysms}

CTA and TOF-MRA are widely available techniques for detection. However, very small aneurysms are often missed. ${ }^{14}$ Computerassisted diagnostics (CAD) acts principally as an internal second 
opinion and improves reading accuracy of physicians. ${ }^{15,16}$ Algorithms have been devised for automatic detections based on MRA/CTA studies using conventional-style and DL methods (On-line Table).

Conventional-Style CAD Systems. The conventional-style CAD systems were based on presupplied characteristics or imaging features, such as vessel curvature, thresholding, or a region-growing algorithm. ${ }^{17}$ The first CAD system geared toward aneurysms reported in the literature was developed by Arimura et $\mathrm{al}^{18}{ }^{18}$ in 2004, which consisted of multiple gray-level thresholding techniques. It showed $100 \%$ sensitivity with 2.4 false-positives (FPs) per patient based on a leave-one-out-by-patient test method. In subsequent validation studies, Hirai et $\mathrm{al}^{19}$ and Kakeda et $\mathrm{al}^{20}$ found that the sensitivity was $100 \%$ and $84 \%$, respectively. However, this algorithm was not fully automated, nor could it detect small or fusiform type of aneurysms. In addition, the aneurysms in these studies were not verified by DSA as an outside reference standard. By using DSA as the reference, Yang et $\mathrm{al}^{17}$ developed a more automated CAD algorithm by combining 2 complementary techniques: 1) automatic intracranial artery segmentation and 2) detection of points of interest from the segmented vessels. They achieved a sensitivity of $95 \%$ with up to 9 additional FP detection marks. However, the sensitivity was lower for small aneurysms $(<5 \mathrm{~mm})$. The conventional-style CAD schemes relied on a similar rulesbased approach in which prior domain knowledge was incorporated into hand-crafted features before using ML techniques as a classifier. The major limitation is that many falsepositive results are found in bending or branching portions of vessels. Multiple methods have been proposed such as an ellipsoid convex enhancement filter to selectively enhance aneurysms while reducing FPs, but the number of FPs remains high. ${ }^{21}$

Deep Learning CAD Systems. More recently, DL-based CAD systems have been developed for aneurysm detection on the basis of MRA. ${ }^{22-24}$ For example, Nakao et $\mathrm{al}^{22}$ used a 2D convolutional neural network to detect aneurysms and reported a sensitivity of $>90 \%$ in a single-center study. Similarly, an opensource neural network has also been applied by using 2D MIPs or original image data. ${ }^{23,24}$ However, generalization of these studies requires further validation. The work by Ueda et $\mathrm{al}^{25}$ subsequently improved the sophistication of a DL methodology with a sensitivity of $91 \%$ and $93 \%$ for the internal and external test datasets, respectively. The external dataset contained images from 4 separate institutions under different environments and MR imaging unit manufacturers, configurations, and field strengths; this feature highlights the rigor and general applicability of the model. However, the study did not have any cases negative for aneurysms. Furthermore, only 74 aneurysms and studies exclusively acquired on Siemens imaging systems were integrated in the external test dataset. Additionally, a high rate of FPs may potentially reduce the enthusiasm of radiologists for the use of this system if distinguishing true-positive aneurysms from FPs becomes too menial.
CTA images have been studied by combining a neural network segmentation model (the HeadXNet model) to augment diagnostic performance in the detection of aneurysms. ${ }^{26}$ In this study, the clinicians showed significant increases in sensitivity, accuracy, and interrater agreement when they were augmented with the model. However, the lack of a reference standard and external data verification, as well as the focus only on nonruptured aneurysms of $>3 \mathrm{~mm}$, limited the generalization and further application of the model.

With respect to the detection of aneurysms, there is a general notion that $\mathrm{CAD}$ algorithms have the potential to shorten reading times and increase radiologists' performances in the laboratory and clinical environment. ${ }^{27}$ Additionally, an AI program of aneurysms may benefit patients who undergo CTA as part of an acute ischemic stroke work-up because it may decrease the likelihood of an incidental aneurysm being undetected. However, to our knowledge, most studies presented so far lack an external reference standard for validation, such as DSA, so further studies are warranted.

\section{Al in the Prediction of Intracranial Aneurysm Rupture Risk}

Automated Morphologic Analysis. Identifying the high-risk morphologic features of saccular aneurysms is very important for rupture-risk stratification and treatment decision. Aneurysm size and shape are regarded as the most important criteria. ${ }^{5}$ In clinical practice, the size is routinely measured manually by physicians on 2D/3D projections. However, manual measurements have inherent limitations of subjectivity and inconsistency, which cause intra- and interobserver variations ${ }^{28}$ and cannot capture the complex geometric features of aneurysms. ${ }^{29}$ Researchers have introduced several computerized procedures to make morphology assessment more objective and consistent. ${ }^{30-32}$ The detection of the neck plane is the key point for multiple subsequent operations. Larrabide et $\mathrm{al}^{30}$ deterministically identified the aneurysm neck based on the topology analysis of the vasculature skeleton and the concepts of deformable cylinders. They can automatically isolate the sac of an aneurysm, reduce interobserver variability, and avoid the bias between the observers. Automatically derived geometric indices were often large, irrespective of segmentation method or operators. ${ }^{31}$

For rapid assessment in the clinical setting, Xiang et $\mathrm{al}^{32}$ devised an image-based vascular analysis toolkit named AView (https:// www.eng.buffalo.edu/Research/Hemo/AView.html) to perform automatic computation of morphologic parameters. AView provided a relatively accurate measurement with an average size error of $0.56 \%$ and volume error of $2.1 \%$ morphologically. ${ }^{33}$ This toolkit also enabled increased consistency in morphologic measurement among operators by $62 \%$ in size and $82 \%$ in neck diameter measurements, which could help potentially avoid inappropriate clinical decisions. In the real-world scenario, Rajabzadeh-Oghaz et $\mathrm{al}^{34}$ from the same group, tested the algorithm on 39 aneurysms and found that the computer-assisted 3D approach can lead to a more accurate and consistent determination of aneurysm size and neck diameter. Besides, DL methods such as the convolutional neural network model developed by Stember et $\mathrm{al}^{23}$ can also be used to automatically analyze morphologic indices. However, there is much work to be done before routine clinical application of these technologies is realized.

AJNR Am J Neuroradiol 41:373-79 Mar 2020 www.ajnr.org 
Automated Calculation of Hemodynamics. Hemodynamics is currently deemed as an important factor for aneurysm formation and rupture risk. ${ }^{35}$ Modern imaging modalities are adequate for the application of computational fluid dynamics modeling. However, complex procedures are time-consuming and demand substantial human interaction, resulting in limited application in real-time clinical practice, which requires automated tools to execute analysis. Seo et $\mathrm{al}^{36}$ developed a highly automated method to execute a computational process with direct use of a voxelized contrast information from 3D angiograms to construct a levelset-based computational "mask" for a hemodynamic simulation. By testing the method in 7 patient-specific cases against the results of manual evaluation by an experienced neurosurgeon, they found that their proposed algorithm was capable of identifying the lesion and connected vessels for various types of aneurysms. The simulation results presented by the algorithm, which include the values and distribution of wall shear stress, were in line with previous computational studies. ${ }^{37}$ However, this study was only hypothesis-generating and requires further refinement and validation. Considering that hemodynamic parameters are critical for the development and rupture risk, such approaches bode rather well for clinical utility due to their automation and diminishing need for human interaction.

Al-Based Prediction of Rupture Risk. An increasing number of unruptured aneurysms are detected with the growing use of advanced imaging techniques. However, we are now confronted with the dilemma of making clinical decisions regarding treatment of unruptured aneurysms, because the risk of treatmentrelated fatality is relatively high, while the rupture risk is low. ${ }^{5,6}$ Predicting rupture risk of aneurysms is challenging and ML is expected to mitigate this problem. Liu et $\mathrm{al}^{38}$ adopted 17 parameters as inputs to a 2-layer feed-forward artificial neural network aimed at predicting the rupture status of anterior communicating artery aneurysms and found an excellent performance. However, this study included only 1 single-center population and used an imbalanced number of samples between ruptured $(n=540)$ and unruptured aneurysms $(n=54)$. The instability of aneurysms is considered a rupture risk. With this knowledge, Liu et $\mathrm{al}^{39}$ applied radiomics tools to extract morphologic features to predict stability and found that flatness was the most important parameter; the area under the curve (AUC) in the testing set reached 0.729 when only flatness was used to predict aneurysm stability, implying the usefulness of radiomics-derived morphologic features for aneurysm rupture risk.

With the introduction of ML methods, it is interesting to understand the distinctive performances of different ML statistical learning approaches. Detmer et $\mathrm{al}^{40}$ and Silva et $\mathrm{al}^{41}$ worked at predicting aneurysm rupture status. They trained several ML methods, including SVM and random forests classifiers. Detmer et al found that multilayer perceptron had the best performance with an AUC of 0.826 (95\% CI, 0.768, 0.883 ) in the test set; important variables included aneurysm location, mean surface curvature, and maximum flow velocity. Silva et al found random forests to have the best performance. In their work, aneurysm location and size were the 2 features that contributed most significantly to the efficacy of the model. This difference may contribute to the uniqueness of input variants and candidate ML methods.

\section{Al in the Prediction of Aneurysm Complications}

Prediction of Complications of SAH. Delayed cerebral ischemia, vasospasm, and cerebral infarction are among the complications of aneurysm rupture; several studies have explored the applications of ML methods to predict at least one of them. Dumont et $\mathrm{al}^{42}$ developed a proof-of-concept artificial neural network prediction model of symptomatic cerebral vasospasm and found the artificial neural network-based model had a better predictive value (AUC of 0.960 ) than 2 multiple logistic regression models $(\mathrm{AUC}=0.933$ and 0.897$)$ developed by Adams et $\mathrm{al}^{43}$ and Qureshi et al, ${ }^{44}$ respectively. Further validation provided an excellent performance in a markedly distinct geographic population setting of southern Arizona with a prospective use of the artificial neural network predictive model. ${ }^{45}$ However, the artificial neural network did not incorporate the timing of symptomatic cerebral vasospasm onset and was not validated in a larger-scale population. Another study had similar findings: ML methods (SVM, random forests, and multilayer perceptron) have a higher performance than logistic regression models in the prediction of delayed cerebral ischemia. ${ }^{46}$

Multiple-task AI has also been studied. Tanioka et $\mathrm{al}^{47}$ used random forests to construct early prediction models of delayed cerebral ischemia, angiographic vasospasm, and cerebral infarction development with clinical variables and matricellular proteins at post-onset days $1-3$. Three such proteins have been reported to be relevant to delayed cerebral ischemia: osteopontin, periostin, and galectin-3. The prediction accuracies of the 3 conditions were $95.1 \%, 78.1 \%$, and $83.8 \%$, respectively. The random forest models found that osteopontin and galectin-3 were among the top 3 most important variables. These novel studies in the assessment of complications of aneurysms have demonstrated excellent performances using ML methods.

Another interesting application is the use of clinical data and CT perfusion from hospital admissions ${ }^{48}$ to predict outcomes of aneurysmal SAH. A random forest model was trained to predict dichotomized mRS ( $\leq 2$ and $>2$ ), and the accuracy was $84.4 \%$ in the training folds and $70.9 \%$ in the validation folds. However, this study had a small population size and therefore cannot be introduced into clinical practice to practically benefit those who have SAH.

Prediction of Treatment Outcomes. Besides aneurysmal SAH, the outcomes of aneurysm treatment have also been explored by ML. For example, the flow diverter has emerged in recent years as one of the endovascular treatments of aneurysms and is particularly suitable for treating wide-neck and intractable aneurysms with unusual morphologies. However, 25\% of treated patients are at high risk for thromboembolism formation and aneurysm rerupture. ${ }^{49}$ Paliwal et al $^{11}$ compared 4 supervised ML algorithms (logistic regression, SVM, K-nearest neighbor, and neural networks) to predict 6-month outcomes of flow diverter-treated aneurysms and found that neural network (AUC $=0.967$ ) performed the best during training; G-SVM (Gaussian-SVM) and neural network had 90\% 


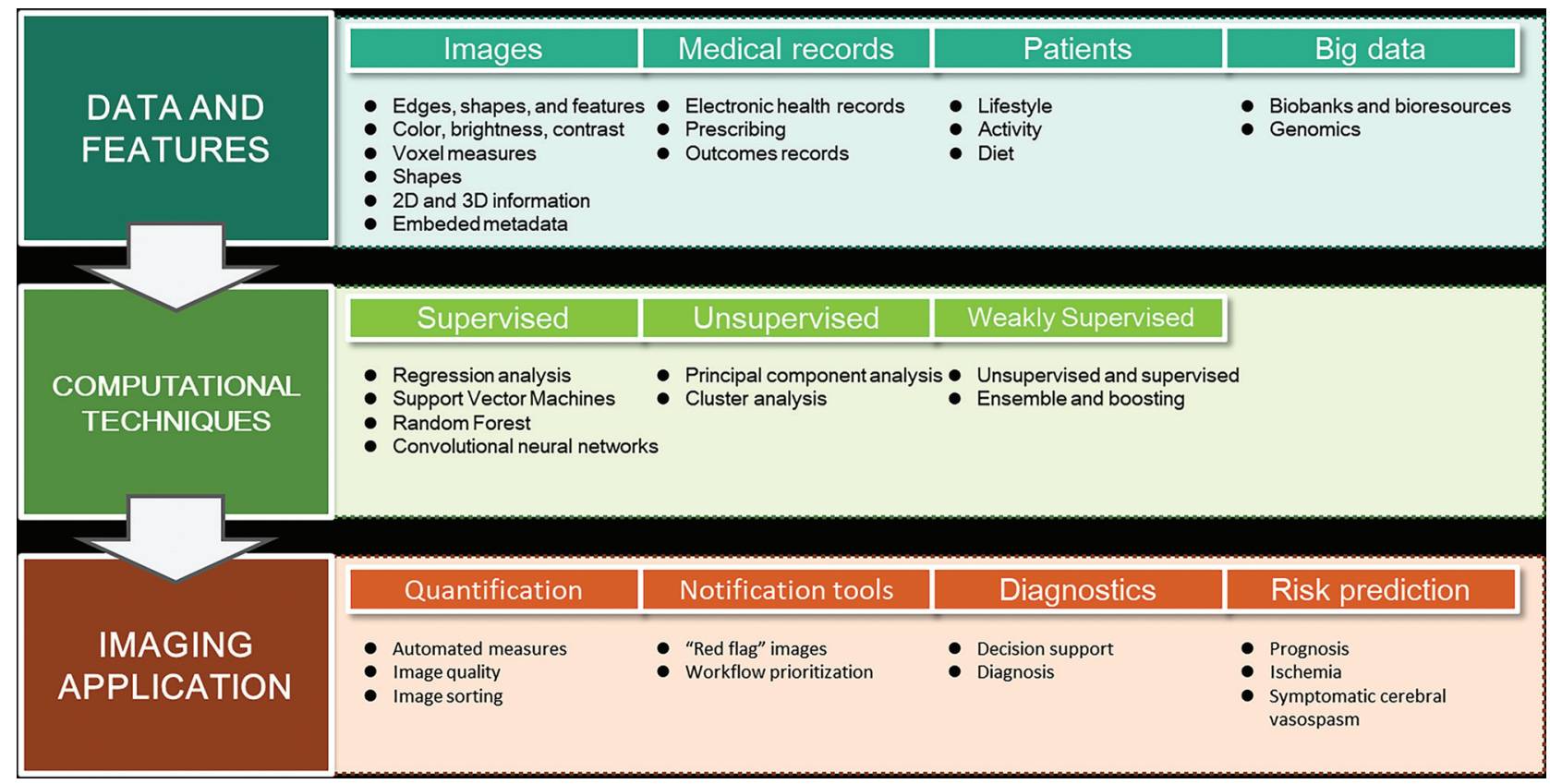

FIG 2. The starting point is high quality data_imaging findings are best contextualized with patients, medical records, and "big data." Computational methods may be supervised, unsupervised, or combined. Imaging applications include quantification, notification tools, and diagnostic and risk predictions. Adapted with permission from Dey et $\mathrm{al}^{10}$ with partial revisions.

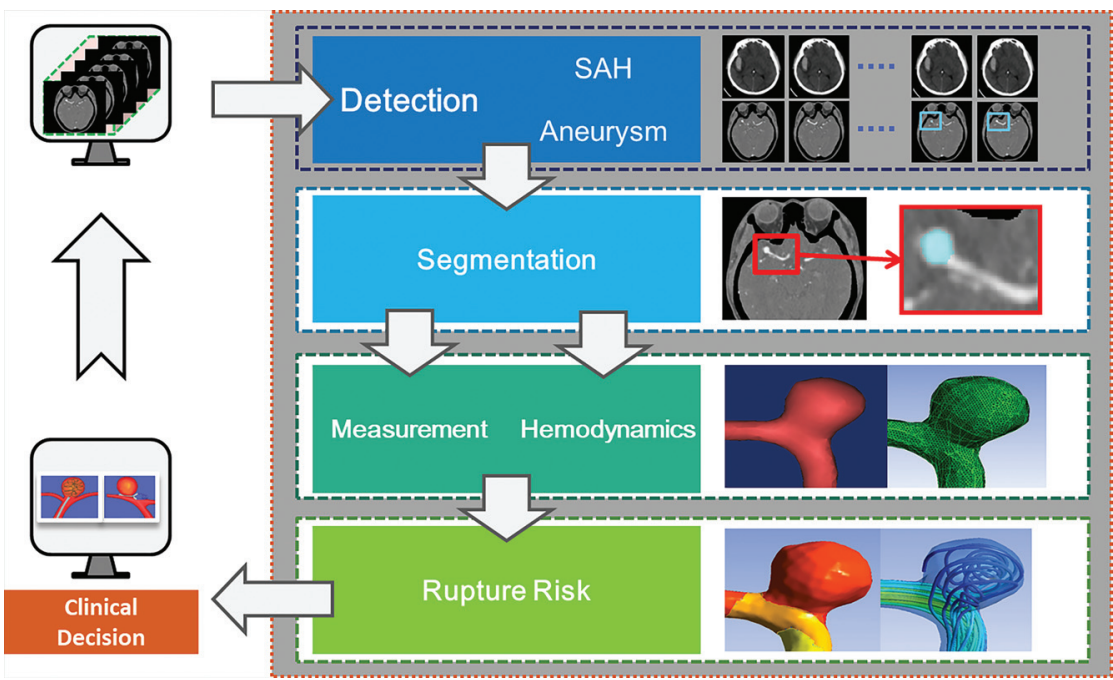

FIG 3. An ideal auto system for intelligent management of intracranial aneurysms. This auto Al system includes the episodes of aneurysm ranging from the detection of SAH and aneurysms to the automatic computation of hemodynamics, dimension index, and clinical decision system.

complications prediction, treatment strategies selection, and risk of recurrence evaluation. AI has been involved in almost all consecutive steps; however, the results are not entirely satisfactory and have some limitations and challenges. First, a systematic review showed no better performance of ML compared with traditional logistic regression in predicting adverse clinical outcomes. ${ }^{50}$ Model validation procedures are often not conducted or well-interpreted; thus, a fair comparison in the domain of real-world case studies is still urgently needed. Second, even though DL methods showed promising potential, there remains considerable debate about the time required to train $\mathrm{DL} .^{51}$ Cost-effectiveness assessment of the high expense of imaging data storage and use of graphics processing units should also

prediction accuracies in the testing cohort. Although a major concern is the absence of an external validation, it is imperative to develop models that help clinicians choose flow-diverter placement in the appropriate patients.

\section{Limitations and Challenges of Al on Aneurysms}

Many investigators have endeavored to develop more intelligent and automated methods to improve the process and streamline the management of aneurysms in clinical practice. Complete management of aneurysms includes detection, rupture risk, be considered. In addition, stakeholders may be responsible for undertaking cost-effectiveness analyses to determine the impact on health care economics, which requires large-scale feasibility studies to evaluate whether unintended consequences may be incurred following the widespread implementation of AI methods. ${ }^{52,53}$ Third, current datasets used for training and validation in aneurysm studies are often small, frequently from only 1 institution, and usually lack external validation within different populations, imaging devices, platforms, and institutions, resulting in algorithm overfitting. 


\section{Future Perspectives of Al in Intracranial Aneurysms}

AI should have a great potential in the management of aneurysms in the future. Potential imaging-based AI applications (Fig 2) mainly contain 4 aspects: quantification, notification tools, diagnostics, and risk prediction for therapy. ${ }^{8}$ For aneurysm management, there are various opportunities for AI, including the following: 1) an automated and reliable prescreening triage system to allow radiologists and emergency physicians to shift their focus to patients at higher risk for adverse events; 2) automated detection and intelligent outcome prediction; 3) subsequent prediction of treatment strategies such as clipping, embolization, or follow-up; and 4) automated and intelligent detection of de novo aneurysm formation or recurrence after treatment and the prediction of rerupture risk.

Researchers should intensify their efforts toward the development of advanced DL algorithms to resolve complex problems, such as extracting information on inflammation of vessel walls from high-resolution vessel wall MR imaging, which has been a nascent area of research in recent years. Additionally, AI tools that can perform multiple tasks, instead of those directed at an isolated problem, are in need. Examples include algorithms that can automatically detect and classify the whole variety of cerebrovascular diseases, including multiple entities like aneurysms, AVMs, and Moyamoya disease. Advanced network structures such as convolutional residual networks, active learning, one-shot learning, and generative adversarial networks may help settle these issues. $^{54}$ These areas are newly emerging and require further investigation.

In our perspective, a successful AI tool for aneurysms is expected to be an integrated toolkit that can intelligently and simultaneously navigate multiple tasks, ranging from detection to prediction (Fig 3). In future work, comprehensive multimodality imaging is worth investigating and is expected to learn and exploit feature representation of images more effectively. In addition, human-in-the-loop AI solutions are quickly emerging concepts toward clinical implementation, which can improve the expert-model performance and act as a gatekeeper for clinical decisions.

Most recently published AI studies have not delineated robust validations of clinical performance and generalizability, including in the area of intracranial aneurysms. Kim et al ${ }^{55}$ recommended 4 criteria for validating the clinical performance of AI algorithms in real-world practice; 1) the external validation must be obtained; 2) using a diagnostic cohort study; 3) arise from multiple institutions; and 4) be performed in a prospective manner. A successful AI tool for aneurysm management must be completely reliable in distinguishing true-positive cases with high confidence, which requires an exceedingly high number of annotated imaging studies, before we can expect widespread implementation in real-world practice.

\section{CONCLUSIONS}

Recent evidence shows that AI, especially deep learning, is rapidly becoming a promising aid in the management of aneurysm. AI is capable of detecting and evaluating rupture risk, triaging clinical therapy strategies, and predicting treatment outcomes. Although we have not quite yet reached the threshold needed for routine clinical application, we believe that $\mathrm{AI}$ is capable of solving these issues in a patient-centric manner but will require advancing network structures and more sophisticated validation processes.

Disclosures: Zhao Shi-RELATED: Grant. Key Projects of the National Natural Science Foundation of China (81830057 for L.J.Z.)* U. Joseph Schoepf-UNRELATED: Other: Dr Schoepf has received institutional research support and/or honoraria for speaking and consulting from Astellas Pharma, Bayer, Bracco, Elucid Biolmaging, GE Healthcare, Guerbet, HeartFlow, and Siemens.* Xiuli Li-RELATED: Key Projects of the National Natural Science Foundation of China (81830057 for LJ.Z.).* Long Jiang ZhangRELATED: Grant: National Key Research and Development Program of China; Key Projects of the National Natural Science Foundation of China, Comments: This work was supported by the National Key Research and Development Program of China (2017YFC0113400 for L.J.Z.) and Key Projects of the National Natural Science Foundation of China $(81830057$ for L.J.Z.).* *Money paid to the institution.

\section{REFERENCES}

1. Vlak MH, Algra A, Brandenburg R, et al. Prevalence of unruptured intracranial aneurysms, with emphasis on sex, age, comorbidity, country, and time period: a systematic review and meta-analysis. Lancet Neurol 2011;10:626-36 CrossRef Medline

2. Li MH, Chen SW, Li YD, et al. Prevalence of unruptured cerebral aneurysms in Chinese adults aged 35 to 75 years: a cross-sectional study. Ann Intern Med 2013;159:514-21 CrossRef Medline

3. Boland GW, Guimaraes AS, Mueller PR. The radiologist's conundrum: benefits and costs of increasing CT capacity and utilization. Eur Radiol 2009;19:9-11 CrossRef Medline

4. McDonald RJ, Schwartz KM, Eckel LJ, et al. The effects of changes in utilization and technological advancements of cross sectional imaging on radiologist workload. Acad Radiol 2015;22:1191-98 CrossRef Medline

5. Morita A, Kirino T, Hashi K, et al; UCAS Japan Investigators. The natural course of unruptured cerebral aneurysms in a Japanese cohort. N Engl J Med 2012;366:2474-82 CrossRef Medline

6. Naggara ON, Lecler A, Oppenheim C, et al. Endovascular treatment of intracranial unruptured aneurysms: a systematic review of the literature on safety with emphasis on subgroup analyses. Radiology 2012;263:828-35 CrossRef Medline

7. He J, Baxter SL, Xu J, et al. The practical implementation of artificial intelligence technologies in medicine. Nat Med 2019;25:30-36 CrossRef Medline

8. Zhou $\mathrm{ZH}$. A brief introduction to weakly supervised learning. Natl Sci Rev 2018;5:45-53 CrossRef

9. LeCun Y, Bengio Y, Hinton G. Deep learning. Nature 2015;521:43644 CrossRef Medline

10. Dey D, Slomka PJ, Leeson P, et al. Artificial intelligence in cardiovascular imaging: JACC state-of-the-art review. J Am Coll Cardiol 2019;73:1317-35 CrossRef Medline

11. Paliwal N, Jaiswal P, Tutino VM, et al. Outcome prediction of intracranial aneurysm treatment by flow diverters using machine learning. Neurosurg Focus 2018;45:E7 CrossRef Medline

12. Varble N, Tutino VM, Yu J, et al. Shared and distinct rupture discriminants of small and large intracranial aneurysms. Stroke 2018;49:856-64 CrossRef Medline

13. Litjens G, Kooi T, Bejnordi BE, et al. A survey on deep learning in medical image analysis. Med Image Anal 2017;42:60-88 CrossRef Medline

14. Bechan RS, van Rooij SB, Sprengers ME, et al. CT angiography versus $3 \mathrm{D}$ rotational angiography in patients with subarachnoid hemorrhage. Neuroradiology 2015;57:1239-46 CrossRef Medline

15. Schalekamp S, van Ginneken B, Koedam E, et al. Computer-aided detection improves detection of pulmonary nodules in chest radiographs beyond the support by bone-suppressed images. Radiology 2014;272:252-61 CrossRef Medline

16. De Fauw J, Ledsam JR, Romera-Paredes B, et al. Clinically applicable deep learning for diagnosis and referral in retinal disease. Nat Med 2018;24:1342-50 CrossRef Medline 
17. Yang X, Blezek DJ, Cheng LTE, et al. Computer-aided detection of intracranial aneurysms in MR angiography. $J$ Digit Imaging 2011;24:86-95 CrossRef Medline

18. Arimura H, Li Q, Korogi Y, et al. Automated computerized scheme for detection of unruptured intracranial aneurysms in three dimensional magnetic resonance angiography. Acad Radiol 2004;11:1093-1104 CrossRef Medline

19. Hirai $T$, Korogi $Y$, Arimura $H$, et al. Intracranial aneurysms at $M R$ angiography: effect of computer-aided diagnosis on radiologists' detection performance. Radiology 2005;237:605-10 CrossRef Medline

20. Kakeda S, Korogi Y, Arimura H, et al. Diagnostic accuracy and reading time to detect intracranial aneurysms on MR angiography using a computer-aided diagnosis system. AJR Am J Roentgenol 2008;190:459-65 CrossRef Medline

21. Jin Z, Arimura H, Kakeda S, et al. An ellipsoid convex enhancement filter for detection of asymptomatic intracranial aneurysm candidates in CAD frameworks. Med Phys 2016;43:951-60 CrossRef Medline

22. Nakao T, Hanaoka S, Nomura Y, et al. Deep neural network-based computer-assisted detection of cerebral aneurysms in MR angiography. J Magn Reson Imaging 2018;47:948-53 CrossRef Medline

23. Stember JN, Chang P, Stember DM, et al. Convolutional neural networks for the detection and measurement of cerebral aneurysms on magnetic resonance angiography. J Digit Imaging 2019;32:80815 CrossRef Medline

24. Sichtermann T, Faron A, Sijben R, et al. Deep learning-based detection of intracranial aneurysms in 3D TOF-MRA. AJNR Am J Neuroradiol 2019;40:25-32 CrossRef Medline

25. Ueda D, Yamamoto A, Nishimori M, et al. Deep learning for MR angiography: Automated detection of cerebral aneurysms. Radiology 2019;290:187-94 CrossRef Medline

26. Park A, Chute C, Rajpurkar P, et al. Deep learning-assisted diagnosis of cerebral aneurysms using the HeadXNet model. JAMA Netw Open 2019;2:e195600 CrossRef Medline

27. Miki S, Hayashi N, Masutani Y, et al. Computer-assisted detection of cerebral aneurysms in MR angiography in a routine image-reading environment: effects on diagnosis by radiologists. AJNR Am J Neuroradiol 2016;37:1038-43 CrossRef Medline

28. Maldaner N, Stienen MN, Bijlenga $\mathrm{P}$, et al. Interrater agreement in the radiologic characterization of ruptured intracranial aneurysms based on computed tomography angiography. World Neurosurg 2017;103:876-82 CrossRef Medline

29. Suh SH, Cloft HJ, Huston J, et al. Interobserver variability of aneurysm morphology: discrimination of the daughter sac. J Neurointerv Surg 2016;8:38-41 CrossRef Medline

30. Larrabide I, Cruz Villa-Uriol M, Cárdenes R, et al. Three-dimensional morphological analysis of intracranial aneurysms: a fully automated method for aneurysm sac isolation and quantification. Med Phys 2011;38:2439-49 CrossRef Medline

31. Piccinelli M, Steinman DA, Hoi Y, et al. Automatic neck plane detection and 3D geometric characterization of aneurysmal sacs. Ann Biomed Eng 2012;40:2188-2211 CrossRef Medline

32. Xiang J, Antiga L, Varble N, et al. AView: An image-based clinical computational tool for intracranial aneurysm flow visualization and clinical management. Ann Biomed Eng 2016;44:1085-96 CrossRef Medline

33. Rajabzadeh-Oghaz H, Varble N, Davies JM, et al. Computer-assisted adjuncts for aneurysmal morphology assessment: toward more precise and accurate approaches. Proc SPIE Int Soc Opt Eng 2017;10134 CrossRef Medline

34. Rajabzadeh-Oghaz H, Varble N, Shallwani H, et al. Computerassisted 3D morphology evaluation of intracranial aneurysms. World Neurosurg 2018;119:e541-50 CrossRef Medline

35. Can A, Du R. Association of hemodynamic factors with intracranial aneurysm formation and rupture: systematic review and meta-analysis. Neurosurgery 2016;78:510-20 CrossRef Medline
36. Seo JH, Eslami P, Caplan J, et al. A highly automated computational method for modeling of intracranial aneurysm hemodynamics. Front Physiol 2018;9:681 CrossRef Medline

37. Cebral JR, Vazquez M, Sforza DM, et al. Analysis of hemodynamics and wall mechanics at sites of cerebral aneurysm rupture. $J$ Neurointerv Surg 2015;7:530-36 CrossRef Medline

38. Liu J, Chen Y, Lan L, et al. Prediction of rupture risk in anterior communicating artery aneurysms with a feed-forward artificial neural network. Eur Radiology 2018;28:3268-75 CrossRef Medline

39. Liu Q, Jiang P, Jiang Y, et al. Prediction of aneurysm stability using a machine learning model based on PyRadiomics-derived morphological features. Stroke 2019;50:2314-21 CrossRef Medline

40. Detmer FJ, Lückehe D, Mut F, et al. Comparison of statistical learning approaches for cerebral aneurysm rupture assessment. Int $J$ Comput Assist Radiology Surg 2019;15:141-50 CrossRef Medline

41. Silva MA, Patel J, Kavouridis V, et al. Machine learning models can detect aneurysm rupture and identify clinical features associated with rupture. World Neurosurg 2019;131:e46-51 CrossRef Medline

42. Dumont TM, Rughani AI, Tranmer BI. Prediction of symptomatic cerebral vasospasm after aneurysmal subarachnoid hemorrhage with an artificial neural network: feasibility and comparison with logistic regression models. World Neurosurg 2011;75:57-63 CrossRef Medline

43. Adams HP Jr, Kassell NF, Torner JC, et al. Predicting cerebral ischemia after aneurysmal subarachnoid hemorrhage: influences of clinical condition, CT results, and antifibrinolytic therapy: a report of the cooperative aneurysm study. Neurology 1987;37:1586-91 CrossRef Medline

44. Qureshi AI, Sung GY, Razumovsky AY, et al. Early identification of patients at risk for symptomatic vasospasm after aneurysmal subarachnoid hemorrhage. Crit Care Med 2000;28:984-90 CrossRef Medline

45. Dumont TM. Prospective assessment of a symptomatic cerebral vasospasm predictive neural network model. World Neurosurg 2016;94:126-30 CrossRef Medline

46. Ramos LA, van der Steen WE, Sales Barros R, et al. Machine learning improves prediction of delayed cerebral ischemia in patients with subarachnoid hemorrhage. J Neurointerv Surg 2019;11:497-502 CrossRef Medline

47. Tanioka S, Ishida F, Nakano F, et al. Machine learning analysis of matricellular proteins and clinical variables for early prediction of delayed cerebral ischemia after aneurysmal subarachnoid hemorrhage. Mol Neurobiol 2019;56:7128-35 CrossRef Medline

48. Rubbert C, Patil KR, Beseoglu K, et al. Prediction of outcome after aneurysmal subarachnoid haemorrhage using data from patient admission. Eur Radiol 2018;28:4949-58 CrossRef Medline

49. Brinjikji W, Murad MH, Lanzino G, et al. Endovascular treatment of intracranial aneurysms with flow diverters: a meta-analysis. Stroke 2013;44:442-47 CrossRef Medline

50. Christodoulou E, Ma J, Collins GS, et al. A systematic review shows no performance benefit of machine learning over logistic regression for clinical prediction models. J Clin Epidemiol 2019;110:12-22 CrossRef Medline

51. Lee JG, Jun S, Cho YW, et al. Deep learning in medical imaging: general overview. Korean J Radiology 2017;18:570-84 CrossRef Medline

52. Retson TA, Besser AH, Sall S, et al. Machine learning and deep neural networks in thoracic and cardiovascular imaging. J Thorac Imaging 2019;34:192-201 CrossRef Medline

53. Morris MA, Saboury B, Burkett B, et al. Reinventing radiology: big data and the future of medical imaging. $J$ Thorac Imaging 2018;33:4-16 CrossRef Medline

54. Hesamian MH, Jia W, He X, et al. Deep learning techniques for medical image segmentation: achievements and challenges. J Digit Imaging 2019;32:582-96 CrossRef Medline

55. Kim DW, Jang HY, Kim KW, et al. Design characteristics of studies reporting the performance of artificial intelligence algorithms for diagnostic analysis of medical images: results from recently published papers. Korean J Radiol 2019;20:405-10 CrossRef Medline 